\title{
(2) OPEN ACCESS \\ Diagnostic criteria for detection of retinal nerve fibre layer thickness and neuroretinal rim width abnormalities in glaucoma
}

\author{
Feihui Zheng, ${ }^{1,2}$ Marco Yu, ${ }^{1,2}$ Christopher Kai-Shun Leung (i) ${ }^{1}$
}

\begin{abstract}
- Additional material is published online only. To view please visit the journal online (http://dx.doi.org/10.1136/ bjophthalmol-2018-313581).
\end{abstract}

${ }^{1}$ Department of Ophthalmology and Visual Sciences, The Chinese University of Hong Kong, Hong Kong, China

${ }^{2}$ Singapore Eye Research Institute, Singapore National Eye Center, Singapore, Singapore

\section{Correspondence to} Dr Christopher Kai-Shun Leung, The Chinese University of Hong Kong, Hong Kong, China; cksleung.cuhk@gmail.com

Received 21 November 2018 Revised 10 March 2019 Accepted 17 April 2019

Published Online First 30 May 2019
Check for updates

(C) Author(s) (or their employer(s)) 2020. Re-use permitted under CC BY-NC. No commercial re-use. See rights and permissions. Published by BMJ.

To cite: Zheng $\mathrm{F}, \mathrm{Yu} \mathrm{M}$, Leung CK-S. Br J Ophthalmol 2020;104:270-275.

\begin{abstract}
Background/aims Although measurements of the Bruch's membrane opening minimum rim width (BMOMRW) and retinal nerve fibre layer thickness (RNFLT) with optical coherence tomography (OCT) have been widely adopted in the diagnostic evaluation of glaucoma, there is no consensus on the diagnostic criteria to define BMO-MRW and RNFLT abnormalities. This study investigated the sensitivities and specificities of different diagnostic criteria based on the OCT classification reports for detection of glaucoma.
\end{abstract}

Methods 340 eyes of 137 patients with glaucoma and 87 healthy individuals, all with axial length $\leq 26 \mathrm{~mm}$, had global and sectoral BMO-MRW and RNFLT measured with Spectralis OCT (Heidelberg Engineering). Six diagnostic criteria were examined: global measurement below the fifth or the first percentile; $\geq 1$ sector measurement below the fifth or the first percentile; superotemporal and/or inferotemporal measurement below the fifth or the first percentile. The sensitivities and specificities of BMO-MRW/RNFLT assessment for detection of glaucoma (eyes with visual field (VF) defects) were compared.

Results Among the six criteria examined, superotemporal and/or inferotemporal measurement below the fifth percentile showed the highest sensitivities and specificities for glaucoma detection. Abnormal superotemporal and/or inferotemporal RNFLT attained a higher sensitivity than abnormal superotemporal and/ or inferotemporal BMO-MRW to detect mild glaucoma (mean VF MD: $-3.32 \pm 1.59 \mathrm{~dB})(97.9 \%$ and $88.4 \%$, respectively, $p=0.006$ ), and glaucoma (mean VF MD: $-9.36 \pm 8.31 \mathrm{~dB})(98.4 \%$ and $93.6 \%$, respectively, $p=0.006)$, at the same specificity $(96.1 \%)$.

Conclusions Superotemporal and/or inferotemporal RNFLT/MRW below the fifth percentile yield the best diagnostic performance for glaucoma detection with RNFLT attains higher sensitivities than MRW at the same specificity in eyes without high myopia.

Clinical diagnosis of glaucoma is predicated on the detection of a thinned retinal nerve fibre layer (RNFL) and a narrowed neuroretinal rim. ${ }^{1}$ While the diagnostic performance of optical coherence tomography (OCT) measurements of the RNFL thickness (RNFLT) and the Bruch's membrane opening derived minimum rim width (BMO-MRW) for detection of glaucoma has been previously reported, ${ }^{2-5}$ it remains unclear whether integrating the assessment of RNFLT and BMO-MRW measurements would improve the diagnostic sensitivity and specificity. One barrier of integrating RNFLT and BMO-MRW assessment is the lack of consensus regarding the criteria to discriminate abnormal from normal RNFLT/BMO-MRW measurements. Previous studies reporting the sensitivity and specificity of RNFLT and BMO-MRW were largely centred on the area under the receiver operating characteristic (ROC) curve (AUC) analysis. ${ }^{2-5}$ The AUC represents a summary measure of a classifier's performance across a range of false-positive rates, typically between $0 \%$ and $100 \%$, against the corresponding true-positive rates. Although the AUC analysis is useful to compare the sensitivities between RNFLT and BMO-MRW at a fixed specificity, detection of BMO-MRW and RNFLT abnormalities in clinical practice rarely refers to the thresholds derived from the ROC curves because the distribution pattern of the ROC curve varies with the demographics (eg, age, refractive errors) and disease characteristics (eg, severity of glaucoma) of the participants recruited in the individual studies. Instead, clinical interpretation of RNFLT and BMO-MRW abnormalities often relies on the diagnostic classification report, which classifies global and sectoral (ie, superotemporal, temporal, inferotemporal, inferonasal, nasal and superonasal) RNFLT and BMO-MRW measurements into three categories: within normal limits (ie, within the normal reference values), borderline (between the first and the fifth percentile of the normal reference values) and outside the normal limits (below the first percentile of the normal reference values), taking reference from the proprietary normative data installed in the OCT instrument. It is an open question in which sector(s) and at what level of statistical significance of RNFLT or BMO-MRW measurements one should consider in the diagnostic evaluation of glaucoma. We set off to identify the optimal criteria to interpret the OCT diagnostic classification report.

\section{METHODS \\ Subjects}

A total of 340 eyes of 224 participants including 188 eyes from 137 patients with glaucoma and 152 eyes from 87 healthy individuals were prospectively and consecutively recruited at the University Eye Center, the Chinese University of Hong Kong and Hong Kong Eye Hospital. All participants received a comprehensive ocular examination and had visual acuity, axial length (IOLMaster; Carl Zeiss Meditec, Dublin, California, USA), refraction and 
intraocular pressure (IOP) (Goldmann applanation tonometry) measured. The neuroretinal rim and the RNFL were imaged by the Spectralis OCT (Heidelberg Engineering, Heidelberg, Germany) with perimetry performed with the Humphrey Field Analyzer II-i (Carl Zeiss Meditec) on the same day. Inclusion criteria were visual acuity $\geq 20 / 40$ and no history of macular disease, neurological disease, refractive or retinal surgery at the time of recruitment. High myopic eyes with an axial length of $>26 \mathrm{~mm}$ were excluded. Patients with glaucoma were consecutively enrolled from the glaucoma clinic. They had glaucoma diagnosed by glaucoma specialists before study enrolment and had visual field (VF) defects confirmed with at least two consecutive examinations in at least one eye. Only the eye with VF defects would be included in the analysis. If both eyes had VF defects, both eyes would be included. The levels of IOP and the OCT measurements of BMO-MRW and RNFLT were not considered as diagnostic criteria. Healthy individuals were enrolled from the general eye clinic. They had no history of IOP $>21 \mathrm{~mm} \mathrm{Hg}$ and no other ocular abnormalities except for mild cataract. The study was conducted in accordance with the ethical standards stated in the 2013 Declaration of Helsinki and approved by the Kowloon Central research ethics committee with written informed consent obtained.

\section{VF examination}

Standard white-on-white automated perimetry (SITA standard 24-2 program) was performed by a masked technician with the Humphrey Field Analyzer II-i (Carl Zeiss Meditec). Included VF tests had fixation losses $\leq 20 \%$ and false-positive errors $\leq 15 \%$. A VF defect had $\geq 3$ non-edge contiguous locations with $\mathrm{p}$ value $<0.05$ (at least one location with $\mathrm{p}<0.01$ ) on the same side of horizontal meridian in the pattern deviation plot confirmed with at least two consecutive examinations.

\section{Neuroretinal rim and RNFL imaging with OCT}

The neuroretinal rim and the RNFL were imaged by a masked technician using the Spectralis OCT (Heidelberg Engineering) with the Glaucoma Module Premium Edition which comprised 24 equally spaced radial B-scans, each with 768 A-scans covering a $15^{\circ}$ region centred on the optic disc for measurement of $\mathrm{BMO}$ MRW, and a $3.5 \mathrm{~mm}$ diameter circle scan for measurement of circumpapillary RNFLT. Twenty-five B-scans were captured and automatically averaged for each B-scan location. All scans were acquired with reference to the subject's specific fovea-BMO (FoBMO) axis. The BMO of each of the 48 meridians and the segmentation of the internal limiting membrane and the posterior boundary of the RNFL of the circle scan were checked and corrected manually if necessary. After excluding 13 eyes with indiscernible BMO for one or more meridians, ${ }^{6}$ and 2 eyes with adhering posterior hyaloid membrane at the optic nerve head obscuring the measurement of BMO-MRW, 188 eyes from 137 patients with glaucoma and 152 eyes from 87 healthy individuals were included. All scans had signal quality $\geq 15$.

\section{Evaluation of diagnostic classification of BMO-MRW and RNFLT for glaucoma detection}

The BMO-MRW and RNFLT were analysed as global and sectoral means, which included the superotemporal $\left(41^{\circ}-80^{\circ}\right)$, superonasal $\left(81^{\circ}-120^{\circ}\right)$, nasal $\left(121^{\circ}-230^{\circ}\right)$, inferonasal $\left(231^{\circ}-270^{\circ}\right)$, inferotemporal $\left(271^{\circ}-310^{\circ}\right)$ and temporal $\left(311^{\circ}-40^{\circ}\right)$ sectors, according to the FoBMO axis. The global and sectoral BMO-MRW and RNFLT measurements were compared with the OCT built-in normative data and classified into within the normal reference range, between the first and the fifth percentile of the normal reference values, or below the first percentile of the normal reference values. Six diagnostic criteria were examined to compare the sensitivities and specificities of BMO-MRW assessment and RNFLT assessment for detection of glaucoma: (1) global measurement below the fifth percentile; (2) global measurement below the first percentile; (3) $\geq 1$ sector of measurement below the fifth percentile; (4) $\geq 1$ sector of measurement below the first percentile; (5) superotemporal and/or inferotemporal measurement below the fifth percentile; (6) superotemporal and/or inferotemporal measurement below the first percentile. The integration of RNFLT assessment and BMO-MRW assessment was performed with an 'or' or 'and' condition on the criterion that showed the highest sensitivity and specificity combination. The sensitivities, as determined by the proportion of eyes meeting a specific diagnostic criterion in the glaucoma group, and the specificities, as determined by the proportion of eyes not meeting a specific diagnostic criterion in the normal group, between RNFLT assessment and BMO-MRW assessment were compared in each of the six diagnostic criteria. The sensitivities and specificities were also compared between integrating RNFLT/BMO-MRW assessment (with an 'or' or 'and' condition) and assessment of RNFLT or BMO-MRW alone.

\section{Statistical analysis}

Statistical analyses were performed with Stata V.14.0 (StataCorp, College Station, Texas, USA). The sensitivities and specificities for discrimination of glaucomatous eyes from normal eyes between BMO-MRW and RNFLT diagnostic classification analyses were compared with the modified Obuchowski's test, which provides adjustment of McNemar's test in the analysis of clustered binary matched-pair data. ${ }^{78}$ The areas under the ROC curve of global and sectoral BMO-MRW and RNFLT measurements were compared with parametric ROC regression after controlling for covariates including age, axial length and BMO area, as well as correlation between fellow eyes. The parametric ROC curve regression model was a probit model, which was a normal cumulative distribution function with input of a linear polynomial in the corresponding quantile function invoked on a false-positive rate. ${ }^{9} 10$ The constant intercept of the polynomial was dependent on the covariates. With an estimated sum of target discordant proportions of 0.1 , the current sample size had a power of $80 \%$ to detect a difference of $0.071,0.064$ and 0.090 in the discordant proportions in the normal group, the glaucoma group and the mild glaucoma group, respectively. Comparisons of biometric parameters between the normal group and the glaucoma group were performed with linear mixed modelling with adjustment of correlation between fellow eyes. P value $<0.05$ was considered statistically significant.

\section{RESULTS}

A total of 340 eyes of 224 participants including 152 eyes of 87 healthy individuals and 188 eyes of 137 patients with glaucoma were included in the study. In the glaucoma group, 95 eyes $(50.5 \%)$ had mild VF defects (VF MD $\geq-6 \mathrm{~dB}$ ), and 93 eyes (49.5\%) had moderate to advanced VF defects (VF MD $<-6$ $\mathrm{dB})$. Patients with glaucoma were older $(58.1 \pm 13.5$ years $)$ and had larger BMO area $\left(2.50 \pm 0.56 \mathrm{~mm}^{2}\right)$ than healthy individuals $\left(53.4 \pm 14.4\right.$ years and $2.31 \pm 0.52 \mathrm{~mm}^{2}$, respectively), but the axial length and spherical equivalent were not significantly different between the groups $(\mathrm{p} \geq 0.160)$ (online supplementary table 1 ). Online supplementary figure 1 shows the sectoral 
Table 1 Sensitivities and specificities of abnormal global BMO-MRW/RNFLT for detection of glaucoma

\begin{tabular}{|c|c|c|c|c|c|c|c|}
\hline & \multicolumn{2}{|c|}{ Global BMO-MRW below the fifth percentile } & \multicolumn{2}{|c|}{ Global RNFLT below the fifth percentile } & \multicolumn{2}{|c|}{ Difference in sensitivities/specificities } & \multirow[t]{2}{*}{$P$ value } \\
\hline & & $95 \% \mathrm{Cl}$ & & $95 \% \mathrm{Cl}$ & & $95 \% \mathrm{Cl}$ & \\
\hline Sensitivity & $78.72 \%$ & $72.10 \%$ to $85.34 \%$ & $88.83 \%$ & $83.69 \%$ to $93.97 \%$ & $-10.11 \%$ & $-15.83 \%$ to $-4.39 \%$ & $<0.001$ \\
\hline \multirow[t]{3}{*}{ Specificity } & $99.34 \%$ & $98.05 \%$ to $100 \%$ & $98.68 \%$ & $96.88 \%$ to $100 \%$ & $0.66 \%$ & $-1.58 \%$ to $2.90 \%$ & 0.565 \\
\hline & \multicolumn{2}{|c|}{ Global BMO-MRW below the first percentile } & \multicolumn{2}{|c|}{ Global RNFLT below the first percentile } & \multicolumn{2}{|c|}{ Difference in sensitivities/specificities } & \\
\hline & & $95 \% \mathrm{Cl}$ & & $95 \% \mathrm{Cl}$ & & $95 \% \mathrm{Cl}$ & \\
\hline Sensitivity & $56.91 \%$ & $49.27 \%$ to $64.56 \%$ & $76.06 \%$ & $69.61 \%$ to $82.52 \%$ & $-19.15 \%$ & $-26.09 \%$ to $-12.21 \%$ & $<0.001$ \\
\hline Specificity & $100.00 \%$ & NA & $99.34 \%$ & $98.05 \%$ to $100 \%$ & $0.66 \%$ & $-0.63 \%$ to $1.95 \%$ & 0.318 \\
\hline
\end{tabular}

BMO-MRW, Bruch's membrane opening-minimum rim width; RNFLT, retinal nerve fibre layer thickness.

BMO-MRW and RNFLT measurements of the glaucoma and the normal groups.

\section{Sensitivities and specificities of BMO-MRW and RNFLT for detection of glaucoma}

Among the six criteria examined (tables 1-3), superotemporal and/or inferotemporal BMO-MRW (table 3, left panel) and superotemporal and/or inferotemporal RNFLT (table 3, middle panel) below the fifth percentile yielded the highest sensitivities and specificities to discriminate glaucomatous eyes ( $\mathrm{n}=188$ eyes) (mean VF MD: $-9.36 \pm 8.31 \mathrm{~dB})$ from normal eyes $(n=152$ eyes). The superotemporal and/or inferotemporal RNFLT below the fifth percentile had a sensitivity of $98.4 \%$ (95\% CI 96.6\% to $100 \%$ ) for the discrimination, which was significantly higher than that of superotemporal and/or inferotemporal BMO-MRW below the fifth percentile $(93.6 \% ; 95 \%$ CI $90.1 \%$ to $97.1 \%)$ $(\mathrm{p}=0.006)$ at the same specificity $96.1 \%$ (table 3$)$. Online supplementary figure 2 shows the Venn diagrams comparing the RNFLT and BMO-MRW assessment.

\section{Sensitivities and specificities of BMO-MRW and RNFLT for detection of mild glaucoma}

We repeated the analyses including eyes with early glaucoma (mean VF MD: $-3.32 \pm 1.59 \mathrm{~dB})(\mathrm{n}=95)$ and normal eyes $(\mathrm{n}=152)$ (online supplementary table 2) using the same sets of diagnostic criteria (online supplementary tables 3-5). Like the analysis including all glaucomatous eyes, the superotemporal and/or inferotemporal RNFLT below the fifth percentile showed the highest sensitivity $(97.9 \%, 95 \%$ CI $95.0 \%$ to $100.0 \%)$ and specificity $(96.1 \%$, 95\% CI $92.1 \%$ to $100.0 \%)$ combination (online supplementary table 5) to detect mild glaucoma among the six criteria examined. The superotemporal and/or inferotemporal BMO-MRW below the fifth percentile had a significantly lower sensitivity $(88.4 \%$, 95\% CI $81.9 \%$ to $95.0 \%)$ than the superotemporal and/or inferotemporal RNFLT below the fifth percentile to detect mild glaucoma at the same specificity. Figure 1 shows two case examples illustrating RNFLT assessment can detect glaucomatous damage missed by BMOMRW assessment. Both patients had inferonasal VF defects with corresponding superotemporal RNFL abnormalities but normal global and sectoral BMO-MRW measurements as shown in the OCT diagnostic classification report.

\section{Comparisons of AUC}

As age, axial length and BMO area are potential confounders of BMO-MRW and RNFLT, ${ }^{11} 12$ parametric ROC regression analysis was performed to evaluate the diagnostic performance of global and sectoral BMO-MRW and RNFLT measurements after controlling for these covariates. Global BMO-MRW showed the greatest AUC (0.978, 95\% CI 0.961 to 0.990$)$ among all the BMO-MRW measurements (the AUC of sectoral BMO-MRW ranged between 0.946 (95\% CI 0.923 to 0.976 ) (the nasal BMO$\mathrm{MRW}$ ) and 0.969 (95\% CI 0.946 to 0.985 ) (the inferotemporal sector)). Likewise, global RNFLT showed the greatest AUC (0.984, 95\% CI 0.966 to 0.995$)$ among all the RNFLT measurements (the AUC of sectoral RNFLT ranged between 0.866 (95\% CI 0.811 to 0.911 ) (the temporal RNFLT) and $0.970(95 \% \mathrm{CI}$ 0.944 to 0.987 ) (the inferotemporal RNFLT)). Whereas there was no significant difference in the AUC between global RNFLT $(0.984,95 \%$ CI 0.966 to 0.995$)$ and global BMO-MRW (0.978, $95 \%$ CI 0.961 to 0.990$)$ for detection of glaucoma $(p=0.454)$, the relative difference in sensitivities between the two classifiers increased with increasing specificity (online supplementary figure 3). The sensitivities of global BMO-MRW and global RNFLT thickness for detection of glaucoma were $94.3 \%$ (95\% CI 89.2\% to $97.8 \%$ ) and $96.4 \%$ (95\% CI $92.8 \%$ to $99.0 \%)$, respectively, at $90.0 \%$ specificity; $88.7 \%$ (95\% CI $80 \%$ to $95.4 \%)$ and $95.2 \%$ ( $95 \%$ CI $87.1 \%$ to $98.9 \%$ ), respectively, at $95.0 \%$ specificity; and $69.9 \%(95 \%$ CI $50.3 \%$ to $88.5 \%)$ and $91.8 \%(95 \% \mathrm{CI}$ $64.9 \%$ to $98.6 \%$ ), respectively, at $99.0 \%$ specificity (table 4 ).

Table 2 Sensitivities and specificities of $\geq 1$ sector of abnormal BMO-MRW/RNFLT for detection of glaucoma

$\geq 1$ sector of BMO-MRW below the fifth percentile

\begin{tabular}{|c|c|c|c|c|c|c|c|}
\hline & \multicolumn{2}{|c|}{ percentile } & \multicolumn{2}{|c|}{$\geq 1$ sector of RNFLT below the fifth percentile } & \multicolumn{2}{|c|}{ Difference in sensitivities/specificities } & \multirow[t]{2}{*}{$P$ value } \\
\hline & & $95 \% \mathrm{Cl}$ & & $95 \% \mathrm{Cl}$ & & $95 \% \mathrm{Cl}$ & \\
\hline Sensitivity & $95.21 \%$ & $92.14 \%$ to $98.28 \%$ & $98.40 \%$ & $96.60 \%$ to $100 \%$ & $-3.19 \%$ & $-6.11 \%$ to $-0.27 \%$ & 0.033 \\
\hline \multirow[t]{2}{*}{ Specificity } & $94.74 \%$ & $90.78 \%$ to $98.69 \%$ & $87.50 \%$ & $81.26 \%$ to $93.74 \%$ & $7.24 \%$ & $0.66 \%$ to $13.82 \%$ & 0.033 \\
\hline & & $95 \% \mathrm{Cl}$ & & $95 \% \mathrm{Cl}$ & & $95 \% \mathrm{Cl}$ & \\
\hline Sensitivity & $83.51 \%$ & $78.10 \%$ to $88.92 \%$ & $95.21 \%$ & $92.13 \%$ to $98.30 \%$ & $-11.70 \%$ & $-17.06 \%$ to $-6.34 \%$ & $<0.001$ \\
\hline
\end{tabular}




\begin{tabular}{|c|c|c|c|c|c|c|c|}
\hline & \multicolumn{2}{|c|}{ ST/IT BMO-MRW below the fifth percentile } & \multicolumn{2}{|c|}{ ST/IT RNFLT below the fifth percentile } & \multicolumn{2}{|c|}{ Difference in sensitivities/specificities } & \multirow[t]{2}{*}{$P$ value } \\
\hline & & $95 \% \mathrm{Cl}$ & & $95 \% \mathrm{Cl}$ & & $95 \% \mathrm{Cl}$ & \\
\hline Sensitivity & $93.62 \%$ & $90.10 \%$ to $97.13 \%$ & $98.40 \%$ & $96.60 \%$ to $100 \%$ & $-4.79 \%$ & $-8.18 \%$ to $-1.39 \%$ & 0.006 \\
\hline \multirow[t]{3}{*}{ Specificity } & $96.05 \%$ & $92.48 \%$ to $99.62 \%$ & $96.05 \%$ & $92.06 \%$ to $100 \%$ & 0 & $-3.67 \%$ to $3.67 \%$ & 1 \\
\hline & ST/IT BM & low the first percentile & ST/IT RN & he first percentile & Difference & ties/specificities & \\
\hline & & $95 \% \mathrm{Cl}$ & & $95 \% \mathrm{Cl}$ & & $95 \% \mathrm{Cl}$ & \\
\hline Sensitivity & $78.19 \%$ & $71.96 \%$ to $84.43 \%$ & $93.62 \%$ & $90.09 \%$ to $97.15 \%$ & $-15.43 \%$ & $-21.56 \%$ to $-9.30 \%$ & $<0.001$ \\
\hline Specificity & $99.34 \%$ & $98.05 \%$ to $100 \%$ & $98.03 \%$ & $95.16 \%$ to $100 \%$ & $1.32 \%$ & $-0.49 \%$ to $3.12 \%$ & 0.157 \\
\hline
\end{tabular}

BMO-MRW, Bruch's membrane opening-minimum rim width; IT, inferotemporal; RNFLT, retinal nerve fibre layer thicknessST, superotemporal.

\section{Integrating RNFLT/BMO-MRW assessment for detection of glaucoma and mild glaucoma}

We then examined whether integrating RNFLT and BMO-MRW assessment with reference to the criterion superotemporal and/ or inferotemporal measurement below the fifth percentile would improve the diagnostic performance to detect glaucoma and mild glaucoma. Integrating RNFLT and BMO-MRW assessment with an 'or' condition did not change the sensitivity and specificity of RNFLT assessment for detection of glaucoma (online supplementary table 6) or mild glaucoma (online supplementary table 7) but increased the sensitivity of BMO-MRW assessment from 93.6\% to $98.9 \%(\mathrm{p}=0.001)$ for detection of glaucoma (online supplementary table 8$)$, and from $88.4 \%$ to $99.0 \%(\mathrm{p}=0.001)$ for detection of mild glaucoma (online supplementary table 9), without compromising the specificity. Integrating BMO-MRW and RNFLT assessment with an 'and' condition did not change the sensitivity and specificity of BMO-MRW assessment for detection of glaucoma (online supplementary table 8 ) or mild

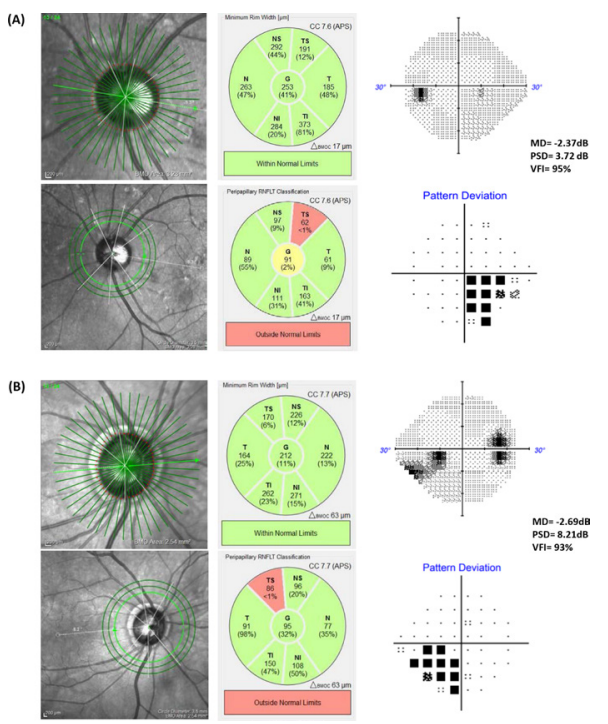

Figure 1 Case examples illustrating diagnostic classification analysis of the retinal nerve fibre layer thickness (RNFLT) can detect glaucomatous damage missed by Bruch's membrane opening-minimum rim width (BM0-MRW). A 51-year-old patient with glaucoma with left inferonasal visual field defects and RNFLT below the first percentile at the superotemporal sector (A), and a 71-year-old patient with glaucoma with right inferonasal visual field defects and RNFLT below the first percentile at the superotemporal sector (B) had global and sectoral BMO-MRW measurements within normal limits. MD, mean deviation; PSD, pattern SD; VFI, visual field index. IN, inferonasal; IT, inferotemporal; N, nasal; SN, superonasal; ST, superotemporal; T, temporal. glaucoma (online supplementary table 9) but decreased the sensitivity of RNFL assessment from $98.4 \%$ to $93.1 \%(p=0.001)$ for detection of glaucoma (online supplementary table 6), and from $97.9 \%$ to $87.4 \%(p=0.001)$ for detection of mild glaucoma (online supplementary table 7 ), without changing the specificity.

\section{DISCUSSION}

To our knowledge, this study provides the first account investigating the criteria to interpret the OCT diagnostic classification reports for detection of glaucoma. Among the six diagnostic criteria examined, superotemporal and/or inferotemporal RNFLT below the fifth percentile exhibited the highest sensitivity and specificity combination to detect glaucoma and mild glaucoma. Surprisingly, BMO-MRW assessment could fail to reveal abnormality even in eyes with confirmed VF defects and RNFL abnormalities (figure 1). Integrating RNFLT assessment to BMO-MRW assessment increased the sensitivity of BMO-MRW assessment without compromising the specificities whereas integrating BMO-MRW assessment to RNFLT assessment did not augment the diagnostic performance. Our finding underscores the importance of RNFL imaging and measurement in the diagnostic evaluation of glaucoma.

Whereas detection of abnormal neuroretinal rim and RNFL measurements in clinical practice frequently relies on OCT diagnostic classification reports, current understanding of the diagnostic performance of BMO-MRW and RNFLT for glaucoma detection is largely derived from the analysis of the ROC curves constructed with reference to the global or sectoral BMO-MRW and RNFLT measurements. ${ }^{2-5}$ In a study comparing the diagnostic performance between BMO-MRW and RNFLT in 50 eyes with preperimetric glaucoma, 50 eyes with perimetric glaucoma and 40 normal eyes, Gmeiner and colleagues showed the AUC to be similar between global BMO-MRW and global RNFLT for discrimination of preperimetric glaucoma $(0.821$ and 0.839 , respectively), and perimetric glaucoma (0.929 and 0.954 , respectively) from normal eyes. ${ }^{3}$ They concluded BMO-MRW and RNFLT to be equally useful for glaucoma detection. In the study by Malik and colleagues examining 74 myopic normal controls and 56 myopic patients with glaucoma, there was no significant difference in AUC between global BMO-MRW (0.900) and global RNFLT (0.897) for the discrimination. ${ }^{4}$ These studies suggest the diagnostic performance of BMO-MRW and RNFLT for detection of glaucoma is comparable. And yet, when we analysed the BMO-MRW and RNFLT diagnostic classification reports, global RNFLT was found to have a significantly higher sensitivity $(76.1 \%-88.8 \%)$ than global BMO-MRW $(56.9 \%$ $78.7 \%)(\mathrm{p}<0.001)$ at similar levels of specificity $(98.7 \%-$ $100.0 \%) \quad(\mathrm{p} \geq 0.318) \quad$ (table 1$)$. The seeming disagreement between the AUC analysis as reported from the previous studies and the diagnostic classification analysis stems from the fact that 
Table 4 Diagnostic performance of global BMO-MRW and global RNFLT for detection of glaucoma using parametric receiver operating characteristic curve regression analysis

\begin{tabular}{|c|c|c|c|c|c|c|c|}
\hline $\begin{array}{l}\text { AUC }(95 \% \\
\text { CI) }\end{array}$ & & $\begin{array}{l}\text { Sensitivity at } \\
90 \% \text { specificity }(95 \% \mathrm{Cl})\end{array}$ & Youden's index & $\begin{array}{l}\text { Sensitivity at } \\
95 \% \text { specificity }(95 \% \mathrm{CI})\end{array}$ & Youden's index & $\begin{array}{l}\text { Sensitivity at } \\
99 \% \text { specificity }(95 \% \mathrm{CI})\end{array}$ & Youden's index \\
\hline BMO-MRW & $0.978(0.961$ to 0.990$)$ & $94.3 \%(89.2 \%$ to $97.8 \%)$ & 0.843 & $88.7 \%(80.0 \%$ to $95.4 \%)$ & 0.837 & $69.9 \%(50.3 \%$ to $88.5 \%)$ & 0.698 \\
\hline RNFLT & $0.984(0.966$ to 0.995$)$ & $96.4 \%(92.8 \%$ to $99.0 \%)$ & 0.864 & $95.2 \%(87.1 \%$ to $98.9 \%)$ & 0.902 & $91.8 \%(64.9 \%$ to $98.6 \%)$ & 0.917 \\
\hline
\end{tabular}

AUC, area under the receiving operator characteristic curve;BMO-MRW, Bruch's membrane opening-minimum rim width; RNFLT, retinal nerve fibre layer thickness.

while the AUCs were typically compared across a wide range of false-positive values (ie, between $0 \%$ and 100\%) and that the sensitivities derived from the ROC curve were often compared at a specificity of $90 \%$ or $95 \%,{ }^{2-5}$ the sensitivities of global BMOMRW and global RNFLT determined from the diagnostic classification analysis were compared at higher specificities (between 98.7\% and 100\%) (table 1). High specificities were observed in the diagnostic classification analysis because the first percentile and the fifth percentile of the normative BMO-MRW/RNFLT data were used to define BMO-MRW/RNFLT abnormalities. Of note, the lack of a significant difference in the AUC between two classifiers does not imply the lack of significant differences in sensitivities across all levels of specificities. In fact, the relative difference in sensitivities derived from the ROC curves between two classifiers can vary widely across a narrow range of specificities. For example, in the study by Chauhan and colleagues, the ROC curves show a higher sensitivity for global BMO-MRW (81\%) than global RNFLT (70\%) (ie, $11 \%$ relative difference in sensitivities) at $95 \%$ specificity for detection of glaucoma. ${ }^{2}$ However, at 99\% specificity, the diagnostic sensitivity becomes higher for global RNFLT (68\%) than global BMO-MRW (18\%) (ie, $-50 \%$ relative difference in sensitivities). Likewise, in the study by Gmeiner and colleagues, although global BMO-MRW and global RNFLT had similar sensitivities $(88 \%$ and $84 \%$, respectively, ie, 4\% relative difference in sensitivities) at $90 \%$ specificity for detection of perimetric glaucoma, global RNFLT showed a higher sensitivity (84\%) than global BMO-MRW (52\%) (ie, $-32 \%$ relative difference in sensitivities) at $95 \%$ specificity. ${ }^{3}$ Performing parametric ROC regression of global BMO-MRW and RNFLT after controlling for age, axial length, BMO area and correlation between fellow eyes in the present study, we also observed global RNFL attained a higher sensitivity than global BMO-MRW, showing an increasing difference in sensitivity with increasing specificity (online supplementary figure 3 ).

The higher diagnostic performance of RNFLT compared with BMO-MRW is observed in global and also in sectoral measurements. Without any consensus regarding how the OCT diagnostic classification report should be interpreted, we investigated six diagnostic criteria for detection of BMO-MRW and RNFLT abnormalities. We examined these criteria instead of reporting the sensitivities and specificities of the individual sectors because clinical interpretation of the diagnostic classification report would unlikely be limited to one individual sector. These criteria facilitate integration of sectoral BMO-MRW/ RNFLT measurements for diagnostic evaluation of glaucoma which would otherwise be difficult to implement in the analysis of the ROC curve. Among the six diagnostic criteria, integrating the superotemporal and inferotemporal sectors yielded the highest sensitivity and specificity combination for detection of RNFLT and BMO-MRW abnormalities. That the superotemporal and/or inferotemporal measurement below the fifth percentile showed the best sensitivity and specificity combination is not unexpected because the superotemporal and the inferotemporal sectors are the most frequent locations where glaucoma develops and progresses. ${ }^{13-16}$ What is worth noting is that similar to the comparison between global BMO-MRW and global RNFLT in which the latter showed a higher sensitivity than the former for detection of glaucoma, abnormal superotemporal and/or inferotemporal RNFLT also exhibited a higher sensitivity than abnormal superotemporal and/or inferotemporal BMO-MRW to detect glaucoma (table 3) and mild glaucoma (online supplementary table 5) at the same specificities. It is also notable that while integrating RNFLT assessment to BMO-MRW assessment increased the sensitivity of BMO-MRW assessment to detect glaucoma (online supplementary table 8) and mild glaucoma (online supplementary table 9), integrating BMO-MRW assessment did not improve the diagnostic performance of RNFLT assessment (online supplementary tables 6 and 7), corroborating the observation that RNFLT is a more sensitive biomarker than BMO-MRW to discriminate glaucomatous from normal eyes. Why does RNFLT assessment outperform BMOMRW assessment even though both structures are essentially composed of the axons of retinal ganglion cells? The stronger association of RNFLT with axon counts, ${ }^{17}$ as well as with visual function, ${ }^{18}$ compared with BMO-MRW may in part explain the better discriminating ability of RNFLT to discern subtle axon loss in early glaucoma. That integrating BMO-MRW assessment to RNFLT assessment does not augment the diagnostic performance for glaucoma detection is also related to the fact that the sensitivities and specificities of RNFLT assessment alone are already high. Nevertheless, our finding does not imply neuroretinal rim assessment is redundant in the diagnostic work-up of glaucoma. Clinical evaluation of the neuroretinal rim is indispensable in the diagnostic evaluation of glaucoma as all forms of optic neuropathies exhibit RNFL thinning. Examination of the neuroretinal rim is important to differentiate glaucomatous from non-glaucomatous optic neuropathies. ${ }^{1}$

One caveat in the interpretation of the diagnostic classification reports is that the current BMO-MRW and RNFLT normative data in the Spectralis OCT were collected from 246 eyes of European descent ${ }^{19}$ whereas all the study participants are Chinese. Nevertheless, it is unlikely that inter-racial differences in BMO-MRW and RNFLT, if any, would have a differential impact on the diagnostic performance between BMO-MRW and RNFLT. Further, the high sensitivities and specificities observed in this study suggest the normative data to be applicable in Chinese for diagnostic evaluation of glaucoma. In a systematic review including over 30 diagnostic accuracy studies of OCT for detection of glaucoma, the best-performing parameter was the inferior RNFLT, which attained a pooled sensitivity of $80 \%$ (95\% CI $68 \%$ to $89 \%$ ) and a pooled specificity of $94 \%$ (95\% CI $85 \%$ to $94 \%) .{ }^{20}$ The diagnostic performance of OCT parameters may vary widely across individual studies because of the different characteristics of the study populations. Diagnostic performance of OCT parameters for glaucoma detection is generally higher in studies including patients with more advanced glaucoma. The high sensitivities observed in the present study is likely related to the fact that glaucoma 
was defined by the presence of VF defects. Glaucomatous eyes exhibiting VF defects likely already have RNFL and/or neuroretinal rim loss. The high specificities are connected to the exclusion of eyes with high myopia. We limited the analysis to eyes with axial length $\leq 26 \mathrm{~mm}$ because the Spectralis OCT and many other commercially available OCT instruments did not include reference data from eyes with high myopia. The superotemporal and inferotemporal RNFL bundles angle decreases with increasing myopia ${ }^{21}$ and the application of the OCT normative data would decrease the specificity for detection of RNFL abnormalities in eyes with myopia. ${ }^{22}$ Another limitation of the Spectralis OCT is the lack of topographic analysis of the RNFLT. The discriminating power of the circumpapillary RNFLT diagnostic classification is inferior to the analysis of RNFL thickness deviation map $\left(6 \times 6 \mathrm{~mm}^{2}\right)$ for glaucoma detection. ${ }^{23}$ It is conceivable that the diagnostic performance of RNFLT assessment can be enhanced with wide-field RNFL imaging. Likewise, the application of the lately investigated BMO-based neuroretinal rim measures such as neuroretinal rim area, ${ }^{24-26}$ neuroretinal rim volume ${ }^{27}$ and number of meridians with abnormal BMO-MRW ${ }^{28}$ may augment the diagnostic performance of BMO-MRW analysis. Prospective studies investigating the longitudinal changes in the RNFLT and neuroretinal rim measurements are required to define their roles in the monitoring of the development and progression of glaucoma.-

Contributors Study design: CK-SL. Analysis: CK-SL, MY, FZ. Manuscript drafting: CK-SL, FZ. Data collection: FZ.

Funding This study was supported by Hong Kong Research Grants Council General Research Fund 2015/2016 (14101215).

Competing interests CK-SL: speaker honorarium—Carl Zeiss Meditec, Heidelberg Engineering; research support—Carl Zeiss Meditec, Heidelberg Engineering.

Patient consent for publication Obtained.

Ethics approval The study was conducted in accordance with the ethical standards stated in the 1964 Declaration of Helsinki and approved by the Kowloon Central Research Ethics Committee.

Provenance and peer review Not commissioned; externally peer reviewed.

Data availability statement Data are available on request.

Open access This is an open access article distributed in accordance with the Creative Commons Attribution Non Commercial (CC BY-NC 4.0) license, which permits others to distribute, remix, adapt, build upon this work non-commercially, and license their derivative works on different terms, provided the original work is properly cited, appropriate credit is given, any changes made indicated, and the use is non-commercial. See: http://creativecommons.org/licenses/by-nc/4.0/.

ORCID iD

Christopher Kai-Shun Leung http://orcid.org/0000-0003-4862-777X

\section{REFERENCES}

1 Weinreb RN, Garway-Heath DF, Leung C, et al. Diagnosis of primary open angle glaucoma. Kugler Publications, 2016.

2 Chauhan BC, O'Leary N, AlMobarak FA, et al. Enhanced detection of open-angle glaucoma with an anatomically accurate optical coherence tomography-derived neuroretinal rim parameter. Ophthalmology 2013;120:535-43.

3 Gmeiner JMD, Schrems WA, Mardin CY, et al. Comparison of Bruch's membrane opening minimum rim width and peripapillary retinal nerve fiber layer thickness in early glaucoma assessment. Invest Ophthalmol Vis Sci 2016:57:0CT575-84.
4 Malik R, Belliveau AC, Sharpe GP, et al. Diagnostic accuracy of optical coherence tomography and scanning laser tomography for identifying glaucoma in myopic eyes. Ophthalmology 2016;123:1181-9.

5 Toshev AP, Lamparter J, Pfeiffer N, et al. Bruch's membrane opening-minimum rim width assessment with spectral-domain optical coherence tomography performs better than confocal scanning laser ophthalmoscopy in discriminating early glaucoma patients from control subjects. J Glaucoma 2017;26:27-33.

6 Zheng F, Wu Z, Leung CKS. Detection of Bruch's membrane opening in healthy individuals and glaucoma patients with and without high myopia. Ophthalmology 2018;125:1537-46.

7 Yang Z, Sun X, Hardin JW. A note on the tests for clustered matched-pair binary data. Biom J 2010;52:638-52.

8 Obuchowski NA. On the comparison of correlated proportions for clustered data. Stat Med 1998;17:1495-507.

9 Janes H, Pepe MS. Adjusting for covariate effects on classification accuracy using the covariate-adjusted receiver operating characteristic curve. Biometrika 2009;96:371-82.

10 Janes $\mathrm{H}$, Longton G, Pepe MS. Accommodating covariates in receiver operating characteristic analysis. Stata J 2009;9:17-39.

11 Araie M, Iwase A, Sugiyama K, et al. Determinants and characteristics of Bruch's membrane opening and Bruch's membrane opening-minimum rim width in a normal Japanese population. Invest Ophthalmol Vis Sci 2017;58:4106-13.

12 Tun TA, Sun C-H, Baskaran M, et al. Determinants of optical coherence tomographyderived minimum neuroretinal rim width in a normal Chinese population. Invest Ophthalmol Vis Sci 2015;56:3337-44.

13 Quigley HA, Miller NR, George T. Clinical evaluation of nerve fiber layer atrophy as an indicator of glaucomatous optic nerve damage. Arch Ophthalmol 1980;98:1564-71.

14 Quigley HA, Addicks EM, Green WR, et al. Optic nerve damage in human glaucoma. II. The site of injury and susceptibility to damage. Arch Ophthalmol 1981;99:635-49.

15 Jonas JB, Fernández MC, Stürmer J. Pattern of glaucomatous neuroretinal rim loss. Ophthalmology 1993;100:63-8.

16 Leung CKS, Choi N, Weinreb RN, et al. Retinal nerve fiber layer imaging with spectral-domain optical coherence tomography: pattern of RNFL defects in glaucoma. Ophthalmology 2010;117:2337-44.

17 Fortune B, Hardin C, Reynaud J, et al. Comparing optic nerve head rim width, rim area, and peripapillary retinal nerve fiber layer thickness to axon count in experimental glaucoma. Invest Ophthalmol Vis Sci 2016;57:0CT404-12.

18 Amini N, Daneshvar R, Sharifipour F, et al. Structure-function relationships in perimetric glaucoma: comparison of minimum-rim width and retinal nerve fiber layer parameters. Invest Ophthalmol Vis Sci 2017;58:4623-31.

19 Spectralis Glaucoma Module Premium Edition User Manual. Software version 6.7 . 2016.

20 Oddone F, Lucenteforte $\mathrm{E}$, Michelessi M, et al. Macular versus retinal nerve fiber layer parameters for diagnosing manifest glaucoma: a systematic review of diagnostic accuracy studies. Ophthalmology 2016;123:939-49.

21 Leung CK-S, Yu M, Weinreb RN, et al. Retinal nerve fiber layer imaging with spectraldomain optical coherence tomography: interpreting the RNFL maps in healthy myopic eyes. Invest Ophthalmol Vis Sci 2012;53:7194-200.

22 Biswas S, Lin C, Leung CKS. Evaluation of a myopic normative database for analysis of retinal nerve fiber layer thickness. JAMA Ophthalmol 2016;134:1032-9.

23 Leung CKS, Lam S, Weinreb RN, et al. Retinal nerve fiber layer imaging with spectraldomain optical coherence tomography: analysis of the retinal nerve fiber layer map for glaucoma detection. Ophthalmology 2010;117:1684-91.

24 Gardiner SK, Ren R, Yang H, et al. A method to estimate the amount of neuroretinal rim tissue in glaucoma: comparison with current methods for measuring rim area. $A m$ J Ophthalmol 2014;157:e541-542:540-9.

25 Enders P, Adler W, Schaub F, et al. Novel Bruch's membrane opening minimum rim area equalizes disc size dependency and offers high diagnostic power for glaucoma. Invest Ophthalmol Vis Sci 2016;57:6596-603.

26 Enders P, Adler W, Schaub F, et al. Optimization strategies for Bruch's membrane opening minimum rim area calculation: sequential versus simultaneous minimization. Sci Rep 2017;7.

27 Fan KC, Tsikata E, Khoueir Z, et al. Enhanced diagnostic capability for glaucoma of 3-dimensional versus 2-dimensional neuroretinal rim parameters using spectral domain optical coherence tomography. J Glaucoma 2017;26:450-8.

28 Danthurebandara VM, Vianna JR, Sharpe GP, et al. Diagnostic accuracy of glaucoma with sector-based and a new total profile-based analysis of neuroretinal rim and retinal nerve fiber layer thickness. Invest Ophthalmol Vis Sci 2016:57:181-7. 